\title{
The secret of sustainable development
}

\author{
Ling Zhang \\ School of The North China Electric Power University,Baoding 071000,China
}

Zhangling940721@126.com

Keywords: TOP-SIS model,PCA model,sustainable development indicators

\begin{abstract}
How to manage increasing population and consumption with the earth's finite resources. How can we do this while at the same time increasing equity and eradicating poverty? In order to reconcile this difficult balance, we construct two models to measure the sustainable capacity of a country.

Defines the method to distinguish more sustainable countries and policies from less sustainable ones.Based on many factors impose impact on the sustainable development, choose PCA (principal component analysis) method to simplify the operation. Improve and optimize the TOP-SIS Model to calculate the relative closeness degree. Base on the conclusion development into five stages, the corresponding closeness of which is used as the standard to measure the sustainable development of a country.
\end{abstract}

\section{Introduction}

How to manage increasing population and consumption with the earth's finite resources. How can we do this while at the same time increasing equity and eradicating poverty? In order to reconcile this difficult balance, the concept of sustainable development was introduced in the 1980's.

Sustainable development is development that meets the needs of the present without compromising the ability of future generations to meet their own needs. It contains within it two key concepts:the concept of needs, in particular the essential needs of the world's poor, to which overriding priority should be given; and the idea of limitations imposed by the state of technology and social organization on the environment's ability to meet present and future needs. ${ }^{[1]}$

In order to define when and how a county is sustainable or unsustainable clearly,we develop two models to measure the sustainable capacity of a country.

\section{Two Models for The Sustainability of a Country}

When obtaining the main indicators as the influence factor, we found too many factors were involved and not convenient for analysis. Therefore, we apply the Principal Components Analysis(PCA)method ${ }^{[2]}$ for evaluation.

\subsection{Model1:TOPSIS}

TOPSIS is based on the concept that the chosen alternative should have the shortest geometric distance from the positive ideal solution and the longest geometric distance from the negative ideal solution ${ }^{[3]}$. Considering the discrepancy between all the countries and their indicators,firstly,we establish a judging matrix.

Then we standardize the judging matrix to unify the data unit and their order of magnitude,and obtain the standard matrix $\mathrm{V}$.

The indicator of "the bigger,the better", we use formula

$v_{i j}=\frac{x_{i j}-\min \left(x_{i j}\right)}{\max \left(x_{j}\right)-\min \left(x_{j}\right)}$

The indicator of "the smaller,the better", we use formula 
$v_{i j}=\frac{\min \left(x_{j}-x_{i j}\right)}{\max \left(x_{j}\right)-\min \left(x_{j}\right)}$

For sake of define the impact of indicators to the degree of sustainable development,we calculate weighted decision matrix $\mathrm{R}$.

The weighted value formulate

$$
r_{i j}=w_{j} \cdot v_{i j}
$$

Where the indicator weighted value is calculated by Entropy Weight Method. ${ }^{[4]}$

We utilize Euclidean Distance Formula ${ }^{[5]}$ to define the degree of sustainable disparity between the actual value and the ideal value.Computing relative closeness degree.

We can measure the level of a country's sustainable development.With the increase of relative closeness degree.the sustainable development ability enhances as well.

In order to make our simulation suitable for all of countries,we measure 28 countries in different degree of sustainable development to define the relationship between relative closeness degree and the countries' sustainability. The consequence is shown in figure:

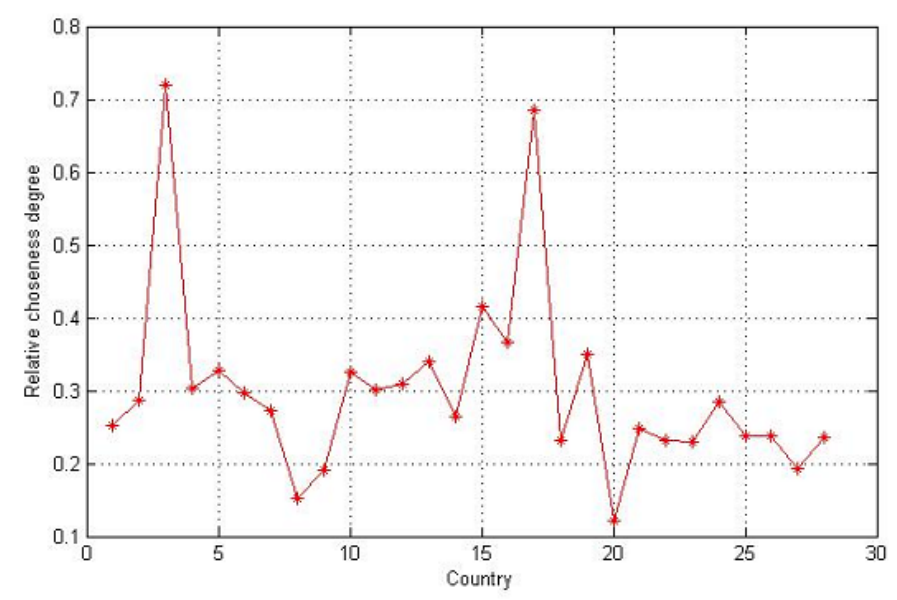

Fig 1: relative closeness degree

These results divide sustainability into 6 stages $^{[6]}$ as,shown in the table as bellow:

\begin{tabular}{|c|l|}
\hline$\eta$ & sustainability \\
\hline $0-0.25$ & unsustainable \\
\hline $0.25-0.35$ & very weak \\
\hline $0.35-0.45$ & weak sustainable \\
\hline $0.45-0.55$ & the preliminary sustainable development \\
\hline $0.55-0.65$ & sustainable development \\
\hline $0.65-0.75$ & the strong sustainable development \\
\hline
\end{tabular}

\subsection{The Phil Haas Model-Based Forecas}

In order to test the rationality of our model, we made an analog emulation to use BP neural network model with Particle Swarm ${ }^{[7]}$ with randomly, choosing 20 countries as analog training samples, and using the remaining 8 countries as analogs, we got the following result: 

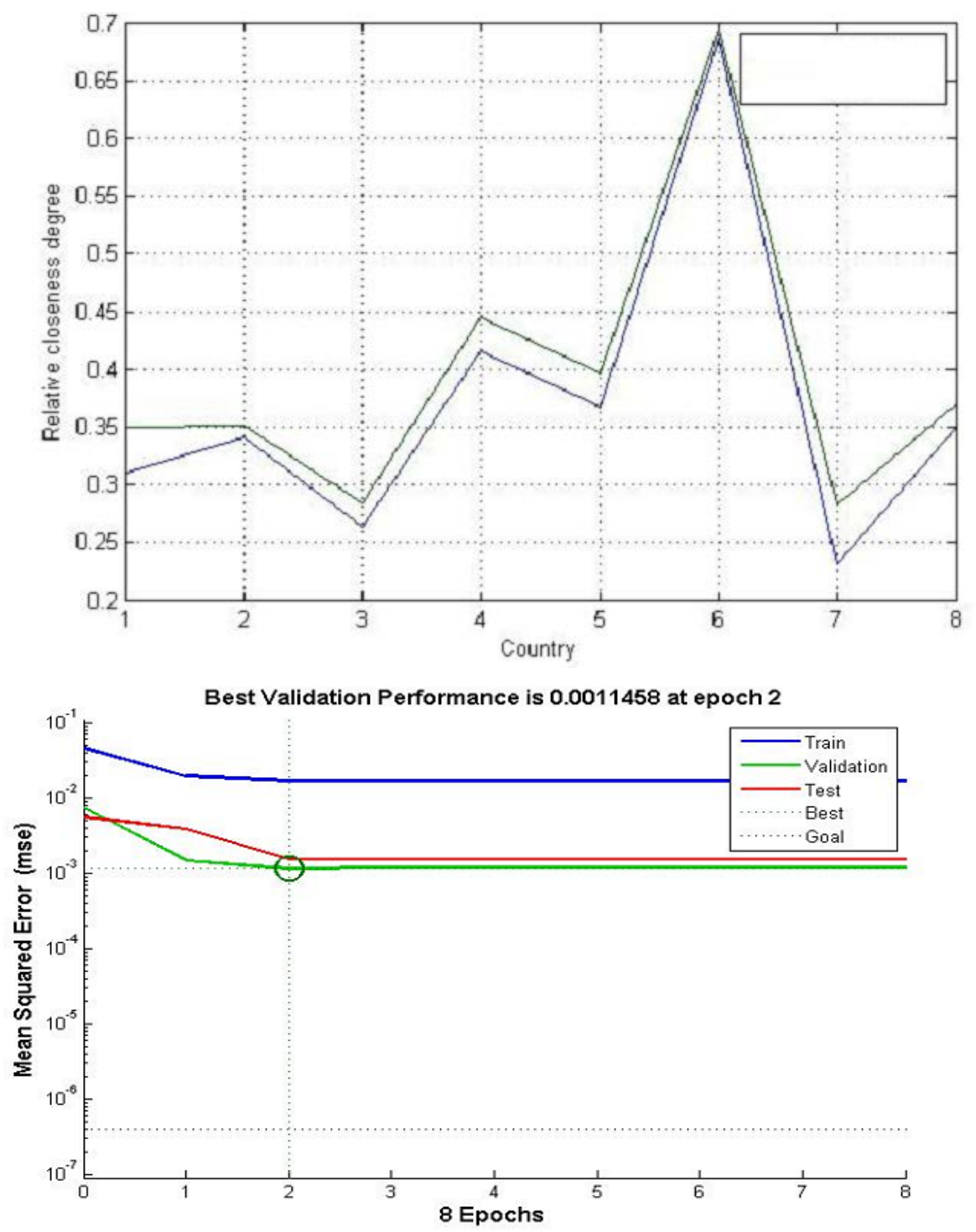

The eight countries in degree of the sustainable development of the two methods are basically identical.TOPSIS Model is proved to be reasonable.

By means of information study, we found there is no way to get all data of a country for recent 20 years. At the same time, the grey prediction model prediction applies GM Model, requiring the data has a strong exponential law, and can only describe the data with single variation law. But there are many factors to influence the sustainable development, and not all the influencing factor is in single changing regulation, so the grey prediction model is impracticable. But the Phil Haas Model can apply to the data with non-single changing regulation, so the Phil Haas Model is better.

For element, we set up a matrix $\mathrm{C}$ to express the same target in different periods for a country by Whitening formula of Phil Haas model ${ }^{[8]}$.

Adding the expected values above of various indicators for a country into the model one to predict the level of the sustainable development of a country in many years.In this way,we can know that the country can be sustainable or not.

\section{Results and analysis}

To test the suitability of our model, residual analysis method is used to calculate the relative error. We defined the predicted value in recent years of a country $\mathrm{C}$ and residual sequence. Then,we get relative error sequence.Finally,we get the Average relative error sequence. When the value is lower than the average relative error,it shows that the model has a high veracity and a good suitability. 


\section{Reference}

[1] A Ma' ckiewicz, W Ratajczak-Computers \& Geosciences, 1993-Elsevier

[2] https://www.iisd.org/sd/

[3] http://en.wikipedia.org/wiki/TOPSIS

[4] http://baike.haosou.com/doc/5994526.html

[5] http://www.cut-the-knot.org/pythagoras/DistanceFormula.shtml

[6] http://wenku.baidu.com/view/eedd60f9770bf78a652954fe.html

[7] http://www.360doc.com/content/13/1005/07/13518188_319082289.shtml

[8] https://excel.ucf.edu/classes/2007/Spring/appsII/Chapter5.pdf 